\title{
Approaches to probiotics properties testing using Caco-2 cells
}

\author{
Viktor Chmykhalo ${ }^{1, *}$, Peter Zolotukhin ${ }^{1}$, Viktor Pakhomov ${ }^{2,3}$, Aleksey Prutskov ${ }^{3}$, Sabina \\ Khairullina ${ }^{4}$, Vladislav Zubtsov ${ }^{3}$, and Marina Egyan ${ }^{3}$ \\ ${ }^{1}$ Southern Federal University, 194/1, Stachki Prospect, 344090, Rostov-on-Don, Russian \\ ${ }^{2}$ Agricultural Research Center «Donskoy», 3, Nauchny gorodok str., Zernograd, Rostov region, \\ Russia \\ ${ }^{3}$ Don State Technical University, 1, Gagarin sq., 344003, Rostov on Don, Russia \\ ${ }^{4}$ Non profit JSC "Zhangir Khan West Kazakhstan Agrarian Technical University", 51, Zhangir khan \\ street, 090009, Uralsk, Kazakhstan
}

\begin{abstract}
Adequate biological models are a prerequisite to screening and development of probiotic drugs. In the present study, Caco-2 cell line is reviewed as a model for analyzing properties of probiotics. This cell culture possesses all the characteristics necessary for evaluating the effects of probiotic drugs on a wide range of both intracellular processes and intercellular interactions. Informativeness of Caco-2 cells is in the focus of the present review.
\end{abstract}

\section{Introduction}

Development of veterinary probiotic drugs for modulating animal health involves a hierarchical set of methods for the probiotic properties testing. The hierarchy of methods is established to balance cost and informativeness at each stage of the analysis, and the most promising candidate agents are promoted to the next stage, while the others are discharged. The first biological level of screening is in vitro testing on cell cultures. The cells used in the tests should be as physiologically close to the final target object as possible, allow the widest possible range of studies to be carried out and, if possible, to create quasi-tissue systems to minimize the need for in vivo studies at the level of screening and selection of candidate agents. In case of eukaryotic models several cell lines meet the criteria, among which the line of human intestinal epithelial cells Caco-2 is leading.

\section{Physiological features of Caco-2 for probiotic research}

Caco-2 cell line is one of the most convenient and frequently used models in studies on effects of various substances and biological agents on the state of intestinal cells, absorption rate, and other parameters [1]. Due to Caco-2 physiological and morphological features, this line allows one to rapidly conduct in vitro screening and predict probable intestinal reactions to the studied agents in vivo [1]. In this view, the same line is even suitable for

\footnotetext{
"Corresponding author: vkchmykhalo@icloud.com
} 
co-culturing and studying the interaction between human intestinal cells and intestinal microbiota and other microorganisms [2]. For example, McWhorter et al. investigated the interaction between bacterial and intestinal cells to determine the colonisation activity of bacteria in the chicken intestines. In this case, the Caco-2 model was used as a confirmatory and screening object [3]. Similar studies were carried out in other laboratories, including those aimed at developing new probiotics for agricultural needs [4].

The main physiological feature of Caco-2, which allows conducting such studies, is changing of their phenotype, when hight confluence level is achieved. They acquire the enterocytes morphology and form tight intercellular contacts, which is also important in the analysis of potential in vivo properties of probiotics [5]. It is this feature that underlies the study of the ability of the probiotic MTCC-5897 to normalize the microbiota in chicken [6].

Moreover, due to this feature, using Caco-2 cells for screening potential of probiotic agents and their derivatives allows to rapidly and efficiently determine acute toxic effects. On one hand, toxic agents cause drastic changes in the general morphology of the confluent Caco-2 culture, and on the other hand, standard methods for evaluating acute toxicity, including trypan blue staining, are applicable for this purpose [7].

\section{Assessment of the effect of probiotics on intracellular processes using flow cytometry and microscopy}

In view of Caco-2 as malignant human cells, their use enables flow cytometry and fluorescence microscopy applications to study their physiological functions under action of probiotics or other biologically active substances (BAS), but also for molecular genetic and systemic biological studies [5] without additional costs.

Regardless of the cell model used (but this is also true for Caco-2), flow cytometry allows testing a multitude of key cellular parameters that quantitatively characterize the effect of BAS and intercellular interactions (between bacteria and intestinal cells). For a sufficiently complete informative assessment of effects, parameters like lysosomal and mitochondrial functioning, lipid content, glucose import rate, cell division rate, generation of reactive oxygen species and cellular thiols are suitable.

Lysosomal function (Lysotracker yellow is an example of the respective molecular probe) is a superposition of the number and activity of lysosomes, which characterizes the endocytosis intensity and cell signaling in response to incoming BAS [8].

Like lysosomal function, mitochondrial function is an integral parameter, which depends on the quantity and activity of mitochondria. A classic example of a dye for analyzing this parameter is Rhodamine 123 [9].

Using Nile red, it is possible to simultaneously determine the content of phospholipids and triglycerides in the cells. These two parameters allow to characterize the intensity of synthetic processes in the cells, as well as the activation level or the pattern of the interaction between the signal pathways such as mTOR, Akt, and HIF1A and, accordingly, indirectly, the intensity of autophagy processes [10].

Investigations of probiotics effects are higlhly informative when using 6-( $\mathrm{N}-(7-$ Nitrobenz-2-oxa-1,3-diazol-4-yl)amino)-6-Deoxyglucose (6-NBDG in short), a nonmetabolizable fluorescent glucose derivative. This physiological parameter has been successfully used in numerous studies, including the one aimed at analyzing interactions between intestinal cells and microflora, in chickens among others [11].

Cell division rate is the most important parameter that characterizes the tissue and organ function of the intestine. One of the most convenient dyes for this purpose is CellTrace CSFE. The study of this physiological parameter is one of the promising methods for studying probiotics properties [12]. 
In the view of the reactive oxygen species (ROS) formed in cells as a messenger of a huge number of cellular signaling systems, studying the pattern of ROS generation in cells allows to estimate a whole range of features of the cell functioning under the influence of various agents. In addition, to date, the influence of microflora and the substances secreted by it on the patterns of the intestinal cell oxidative status systems has been well characterized [13]. When conducting flow cytometry, two dyes that are used together are the most convenient - a molecular probe for ROS in cytosol - CellRox Orange - and a molecular probe for ROS in nuclei and mitochondria - CellRox Green.

Cellular thiols act as an additional cellular parameter characterizing both the functioning of metabolic systems (for example, the relative activation degree of glycolysis and the pentose-phosphate shunt) and cellular signaling in oxidative status control systems (when tested together with the indicated ROS probes). An example of a suitable probe for flow cytometry is ThiolTracker Violet, which allows quantitative detection of cell thiols, mostly represented by glutathione. This parameter was valuable in studies of intestinal physiology depending on the state of microflora [14].

Since the above mentioned molecular probes almost cover the entire visible spectrum, to gate living cells in low cytometry one needs to use two corresponding dyes - 7-AAD and Sytox Blue. The use of these dyes makes it possible to carry out a multiparametric analysis of the state of cells and, without any significant interference, to study up to 4 parameters simultaneously in the same cell [15].

Some of the listed parameters can be analyzed not only by flow cytometry, followed by quantitative data, but also by fluorescence microscopy. In this case, it is possible to detect changes in the quality parameters - changes in the morphology of cells, organelles, and their location in the cell [16].

For example, in case of mitochondria, using MitoTracker Red (a more photostable dye than Rhodamine 123) allows to analyse both the trans-membrane potential (semiquantitatively using optical densitometry), localization of mitochondria in the cell and their morphology. Such changes are detected, including when the cells are exposed to derivatives of probiotic cultures [17].

Using immunocytochemistry and genetic engineering marker CellLight PeroxisomeGFP, studying the peroxisomes localization is possible. A change in the peroxisomal system function is an in vitro marker of the biochemical characteristics of probiotic cultures, as shown, for example, for butyrate producers [18].

Using the above mentioned LysoTracker Yellow dye and immunocytochemical staining of the protein MAP1LC3B (membrane protein of mature autophagosomes) allows to study autophagy flux. Autophagy modulation is one of the established mechanisms of antiinflammatory effects of probiotics and normalization of the intestinal mucosa [19].

Additionally, using immunocytochemistry, one more important function of the intestine is studied - the ability to import amino acids. For example, protein SLC3A2 (CD98), localized on the surface of cells and thus allowing immunocytochemical staining without fixating and permeabilizing the cells, is a component of several amino acids importing complexes into cells. Expression of this protein is dependent on the functional status of intestinal cells and intercellular signaling in the intestine [20].

The discussed fluorescent dyes used to assess the effects of probiotics on Caco-2 cellular proccesses are summarized in Table 1. 
Table 1. The informative cell parameters for characterising of probiotics influence and comparable fluorescent dyes.

\begin{tabular}{|l|l|l|}
\hline \multicolumn{1}{|c|}{ Cell parameter } & \multicolumn{1}{|c|}{ Fluorescent dyes } & Reference \\
\hline Lysosomal function & Lysotracker yellow & {$[8]$} \\
\hline Mitochondrial function & Rhodamine 123, MitoTracker Red & {$[9],[17]$} \\
\hline $\begin{array}{l}\text { The content of phospholipids and } \\
\text { triglycerides }\end{array}$ & Nile Red & {$[10]$} \\
\hline The glucose uptake & 6-NBDG & {$[11]$} \\
\hline Proliferation rate & CellTrace CSFE & {$[12]$} \\
\hline ROS level & CellRox Orange, CellRox Green & {$[13]$} \\
\hline Cellular thiols & ThiolTracker Violet & {$[14]$} \\
\hline Viability & 7-AAD, Sytox Blue & {$[15]$} \\
\hline The peroxisomal system function & CellLight Peroxisome-GFP & {$[18]$} \\
\hline Autophagy intensity & $\begin{array}{l}\text { LysoTracker Yellow, immunostaning } \\
\text { against MAP1LC3B }\end{array}$ & {$[19]$} \\
\hline Amino acids import rate & Immunostaning against SLC3A2 & {$[20]$} \\
\hline
\end{tabular}

\section{Analysis of the effects of probiotics on molecular pathways}

Immunocytochemistry methods also allow to evaluate signal pathways function - and this technology is highly informative when combined with molecular genetic studies on the markers expression. For example, in the central antioxidant and xenobiotic defense pathway NFE2L2/AP-1 (also referred to in the literature as the NRF2 and AP-1 pathway), there are several sensor proteins that change cell localization and control transcription of the effector genes. In a study, the effect of probiotics on this pathway was studied on Caco-2 cells [21].

In this case, immunocytochemistry detects changes in the compartmentalisation of the sensory component of the pathway, and molecular genetics reveals shifts in the expression of the effector molecules and even in the functional status of individual cell compartments. For example, the differential expression of the XBP1 gene transcripts 1 and 2 allows one to characterize the functioning of the endoplasmic reticulum. Normalization of the EPR activity is also one of the important effects of probiotics during their screening [22].

Molecular genetic methods are also most convenient for screening the immune modulating properties of probiotics and avoid the use of co-culture technology for such studies. The key pathways to study in this case are the pathway of nuclear factor kappa B (NF-kappaB) and hypoxia-inducible factor HIF1A. The NF-kappaB pathway directly controls the expression of cytokine proteins and other agents of intracellular and intercellular interactions [23]. At the same time, the HIF1A pathway adjacent and interdependent with the previous one controls not only angiogenesis and reactions to oxygen level fluctuations, but also the process of autophagy, the nature of energy metabolism, cell proliferation and is responsible for maintaining the intestinal crypt stem cell niche [24] and functioning of a system of pathways maintaining stem cells status and providing epithelial-mesenchymal transition [25]. 


\section{Conclusions}

To analyze the properties of probiotic microorganisms and BAS synthesized by such organisms, a wide range of molecular and cellular biology methods is available today from studies of intestinal cell morphology in vitro and to molecular genetic analysis in vitro and in vivo (Figure 1).

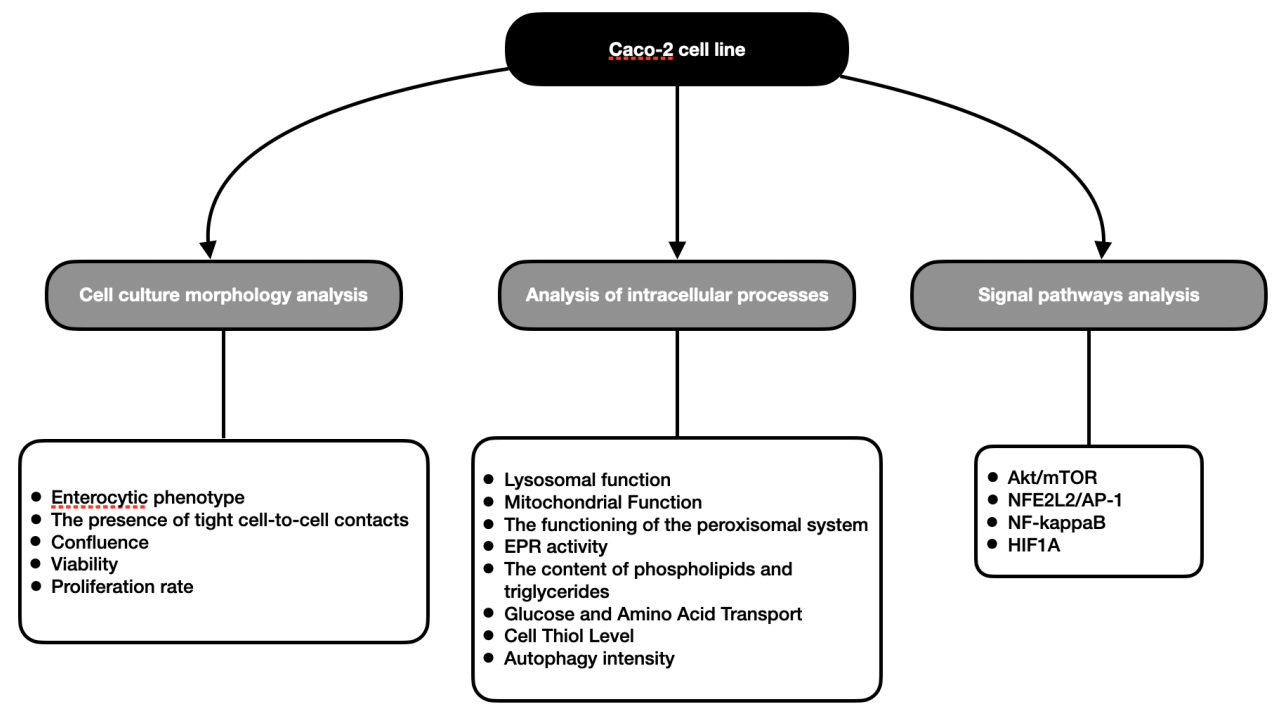

Fig. 1. Key assays using Caco-2 cell culture as a model for studying the effects of probiotics.

The range of research models for such experiments is also wide - from artificial systems and cell cultures with morphological signs of enterocytes to ex vivo and in vivo objects. As noted above, Caco-2 cells appear to be one of the most appropriate models for this area of investigation.

\section{Acknowledgements}

Authors acknowledge the support of the Government of the Russian Federation (contract No. 075-15-2019-1880).

The authors declare no conflict of interest.

\section{References}

1. X. Cai, X. Chen, N. Yin, H. Du, G. Sun, L. Wang, Y. Xu, Y. Chen, Y. Cui, Food Funct 8, 4592-4600 (2017) doi: 10.1039/c7fo01348e

2. M. Gagnon, A. Zihler Berner, N. Chervet, C. Chassard, C. Lacroix, J Microbiol Methods 94, 274-279 (2013) doi: 10.1016/j.mimet.2013.06.027

3. A.R. McWhorter, R. Tearle, T.S. Moyle, K.K. Chousalkar, Vet Res 50, 71 (2019) doi: 10.1186/s13567-019-0688-1

4. Y. Xu, Y. Tian, Y. Cao, J. Li, H. Guo, Y. Su, Y. Tian, C. Wang, T. Wang, L. Zhang, Front Physiol 10, ID 937 (2019) doi: 10.3389/fphys.2019.00937

5. B. Yao, J. He, X. Yin, Y. Shi, J. Wan, Z. Tian, Toxicol Lett 316, 109-118 (2019) doi: 10.1016/j.toxlet.2019.08.024 
6. M. Iqbal Bhat, K. Sowmya, S. Kapila Rajeev Kapila, Probiotics Antimicrob Proteins (2019) doi: 10.1007/s12602-019-09608-8

7. A. Saber, B. Alipour, Z. Faghfoori, A. Mousavi Jam, A. Yari Khosroushahi, Nutr. Res. 41, 36-46 (2017) doi: 10.1016/j.nutres.2017.04.001

8. S. Chikte Neelam Panchal, G. Warnes, Cytometry A 85, 169-178 (2014) doi: 10.1002/cyto.a.22312

9. B. Chazotte, Cold Spring Harb Protoc 7, 892-894 (2011) doi: 10.1101/pdb.prot5648

10. J.G. Schnitzler, S.J. Bernelot Moens, F. Tiessens, G.J. Bakker, G.M. DallingaThie, A.K. Groen, M. Nieuwdorp, E.S.G. Stroes, J. Kroon, J. Lipid Res. 58, 2210-2219 (2017) doi: 10.1194/jlr.D073197

11. F.T. Lim, S.M. Lim, K. Ramasamy, Benef Microbes 8, 97-109 (2017) doi: 10.3920/BM2016.0048

12. M.F. Laursen, R.P. Laursen, A. Larnkjær, K.F. Michaelsen, M.I. Bahl, T.R. Licht, BMC Microbiol 17, 175 (2017) doi: 10.1186/s12866-017-1090-7

13. R.M. Jones, A.S. Neish, Free Radic. Biol. Med. 105, 41-47 (2017) doi: 10.1016/j.freeradbiomed.2016.10.495

14. A. Mardinoglu, S. Shoaie, M. Bergentall, P. Ghaffari, C. Zhang, E. Larsson, F. Bäckhed, J. Nielsen, Mol. Syst. Biol. 11, 834 (2015) doi: 10.15252/msb.20156487

15. C.W. Nuelle, C.R. Cook, A.M. Stoker, J.L. Cook, S.L. Sherman, J. Orthop Translat 8, 20-24 (2016) doi: 10.1016/j.jot.2016.08.002

16. M.-H. Schuler, A. Lewandowska, G. Di Caprio, W. Skillern, S. Upadhyayula, T. Kirchhausen, J.M. Shaw, B. Cunniff, Mol. Biol. Cell. 28, 2159-2169 (2017) doi: 10.1091/mbc.E16-10-0741

17. C. Xu, L. Qiao, L. Ma, Y. Guo, X. Dou, S. Yan, B. Zhang, A. Roman, Int. J. Nanomedicine 14, 4491-4502 (2019) doi: 10.2147/IJN.S199193

18. H. Weng, K. Endo, J. Li, N. Kito, N. Iwai, PLoS One 10, e0117851 (2015) doi: 10.1371/journal.pone.0117851

19. M. Zaylaa, J. Alard, I. Al Kassaa, V. Peucelle, D. Boutillier, J. Desramaut, P. Rosenstiel, H.T.T. Nguyen, F. Dabboussi, B. Pot, C. Grangette, Cell Physiol. Biochem. 53, 774-793 (2019) doi: 10.33594/000000172

20. H. Thi Thu Nguyen, G. Dalmasso, L. Torkvist, J. Halfvarson, Y. Yan, H. Laroui, D. Shmerling, T. Tallone, M. D'Amato, S.V. Sitaraman, D. Merlin, J. Clin. Invest. 121, 1733-1747 (2011) doi: 10.1172/JCI44631

21. G. Mu, H. Li, Y. Tuo, Y. Gao, Y. Zhang, J. Dairy Sci. 102, 863-6875 (2019) doi: 10.3168/jds.2019-16447

22. A. Kishino, K. Hayashi, C. Hidai, T. Masuda, Y. Nomura, T. Oshima, Sci. Rep. 7, 4442 (2017) doi: 10.1038/s41598-017-02960-1

23. D.-E. Kim, J.-K. Kim, S.-K. Han, S.-E. Jang, M. Joo Han, D.-H. Kim, J. Med. Food 22, 1022-1031 (2019) doi: 10.1089/jmf.2019.4419

24. Y. Chen, S.-H. Lee, Y.-H. Tsai, S.-H. Tseng, J. Surg. Res. 187, 85-93 (2014) doi: 10.1016/j.jss.2013.10.001

25. D. Vergara, P. Simeone, M. Damato, M. Maffia, P. Lanuti, M. Trerotola, J. Oncol. 2019, 1253727 (2019) doi: 10.1155/2019/1253727 